\title{
CD4 testing after initiation of antiretroviral therapy: Analysis of routine data from the South African HIV programme
}

\begin{tabular}{|c|c|}
\hline \multicolumn{2}{|c|}{ 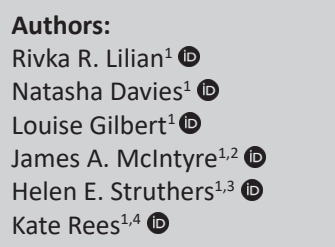 } \\
\hline \multicolumn{2}{|c|}{$\begin{array}{l}\text { Affiliations: } \\
{ }^{1} \text { Anova Health Institute, } \\
\text { Johannesburg, South Africa }\end{array}$} \\
\hline \multicolumn{2}{|c|}{$\begin{array}{l}{ }^{2} \text { School of Public Health and } \\
\text { Family Medicine, University } \\
\text { of Cape Town, Cape Town, } \\
\text { South Africa }\end{array}$} \\
\hline \multicolumn{2}{|c|}{$\begin{array}{l}{ }^{3} \text { Division of Infectious } \\
\text { Diseases and HIV Medicine, } \\
\text { Department of Medicine, } \\
\text { University of Cape Town, } \\
\text { Cape Town, South Africa }\end{array}$} \\
\hline \multicolumn{2}{|c|}{$\begin{array}{l}{ }^{4} \text { Department of Community } \\
\text { Health, School of Public } \\
\text { Health, University of the } \\
\text { Witwatersrand, Johannesburg, } \\
\text { South Africa }\end{array}$} \\
\hline \multicolumn{2}{|c|}{$\begin{array}{l}\text { Corresponding author: } \\
\text { Kate Rees, } \\
\text { rees@anovahealth.co.za }\end{array}$} \\
\hline $\begin{array}{l}\text { Dates: } \\
\text { Received: } 04 \mathrm{~S} \\
\text { Accepted: } 05 \mathrm{C} \\
\text { Published: } 14\end{array}$ & $\begin{array}{l}\text { Sept. } 2020 \\
\text { Oct. } 2020 \\
\text { Dec. } 2020\end{array}$ \\
\hline \multicolumn{2}{|c|}{$\begin{array}{l}\text { How to cite this article: } \\
\text { Lilian RR, Davies N, Gilbert L, } \\
\text { et al. CD4 testing after } \\
\text { initiation of antiretroviral } \\
\text { therapy: Analysis of routine } \\
\text { data from the South African } \\
\text { HIV programme. S Afr J HIV } \\
\text { Med. } 2020 ; 21(1) \text {, a1165. } \\
\text { https://doi.org/10.4102/ } \\
\text { sajhivmed.v21i1.1165 }\end{array}$} \\
\hline \multicolumn{2}{|l|}{ Read online: } \\
\hline 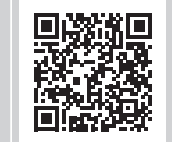 & $\begin{array}{l}\text { Scan this QR } \\
\text { code with your } \\
\text { smart phone or } \\
\text { mobile device } \\
\text { to read online. }\end{array}$ \\
\hline
\end{tabular}

Background: People living with HIV (PLHIV) who have low CD4 counts require advanced clinical care (ACC) to minimise morbidity and mortality risk. These patients include immunological non-responders (INRs) with low CD4 counts despite a suppressed viral load.

Objectives: To determine the proportion of patients with low CD4 counts after antiretroviral therapy (ART) initiation and to describe INRs within that group.

Methods: Routine Three Interlinked Electronic Registers.Net (TIER.Net) data from four South African districts were analysed for adult PLHIV on ART $>12$ months. Immunological nonresponders were defined as patients on ART $>4$ years who were virally suppressed (viral load $<1000$ copies $/ \mathrm{mL}$ ) with a CD4 count $\leq 350 \mathrm{cell} / \mathrm{mm}^{3}$.

Results: Baseline CD4 was recorded for $80.9 \%$ of the 869571 patients newly initiating ART, with $37.2 \%$ of those starting ART since 2017 having baseline counts $\leq 200$ cells $/ \mathrm{mm}^{3}$. Amongst all 1178190 patients on ART, only 46.5\% had a CD4 test after ART initiation and of these, $14.3 \%$ had CD4 $\leq 200$ cells $/ \mathrm{mm}^{3}$. This proportion was highest amongst patients on ART $\leq 2$ years $(19.7 \%)(p<0.001)$. Amongst virally suppressed patients, $20.0 \%$ were INRs. Immunological non-response was significantly more likely amongst patients on second-line ART (adjusted odds ratio [aOR] 1.79), those aged $35-45$ and $\geq 45$ years (aOR 1.15 and 1.50 , respectively), males (aOR 2.28) and patients with confirmed TB (aOR 2.49), and was significantly less likely in cases with higher baseline CD4 count (aOR 0.35).

Conclusion: CD4 testing subsequent to ART initiation is poorly implemented and there is a notable proportion of patients with low CD4 counts. Guidelines regarding CD4 testing and ACC need to be more widely implemented to identify patients with low CD4 counts and improve their outcomes.

Keywords: CD4; HIV; South Africa; advanced clinical care; immunological non-responder; TIER.Net.

\section{Introduction}

Antiretroviral therapy (ART) improves outcomes of people living with human immunodeficiency virus (PLHIV) by reducing HIV viral load (VL), which enables immune recovery, including recovery of CD4 count. Where patients have CD4 counts below 200 cells $/ \mathrm{mm}^{3}$, known as advanced HIV disease, advanced clinical care (ACC) should be provided to improve patient outcomes, including screening, management of opportunistic infections and focussed adherence support. ${ }^{1}$ Advanced clinical care is particularly important in South Africa, considering that up to $40 \%$ of adults initiate ART with CD4 $<200$ cells $/ \mathrm{mm}^{3}$. $^{2}$ Furthermore, up to $50 \%$ of patients receiving ART demonstrate poor CD4 recovery, dependant on multiple factors including baseline CD4 count and CD4 recovery definition. ${ }^{3,4,5,6}$ In some patients, known as immunological non-responders (INRs), CD4 count remains low despite a suppressed VL, which significantly increases the risk of mortality. ${ }^{7}$

South African guidelines include baseline CD4 testing for ART patients to assess cotrimoxazole prophylaxis (CPT) eligibility (where CD4 count is $\leq 200$ cells $/ \mathrm{mm}^{3}$ ) and to determine susceptibility to opportunistic infections. ${ }^{8} \mathrm{CD} 4$ monitoring should be repeated 12 months after ART initiation, 6 monthly until CPT eligibility ceases, 6 monthly if VL increases above 1000 copies $/ \mathrm{mL}$ and if a patient requires re-initiation onto ART. ${ }^{8}$ Such CD4 testing is critical to ensure correct ACC Copyright: @ 2020. The Authors. Licensee: AOSIS. This work is licensed under the Creative Commons Attribution License. 
management for patients with low CD4 counts to mitigate higher morbidity and mortality risks.

The South African ART programme is routinely managed using an electronic database known as Three Interlinked Electronic Registers.Net (TIER.Net). This study analysed routine programmatic TIER.Net data to assessimplementation of post-ART CD4 testing and occurrence of poor CD4 recovery in order to highlight areas for intervention to improve CD4 monitoring and subsequent patient management. Specifically, this study aimed to: (1) calculate the proportion of patients on ART with CD4 tests subsequent to ART initiation, (2) describe the proportion of patients with subsequent CD 4 counts $\leq 200$ and $\leq 350$ cells $/ \mathrm{mm}^{3}$ by ART duration and (3) assess the proportion of INRs and describe their characteristics.

\section{Methods}

\section{Data source and study population}

Three Interlinked Electronic Registers.Net data were extracted in March 2020 for Johannesburg and Sedibeng districts in Gauteng province and Capricorn and Mopani districts in Limpopo province. These districts were chosen as a convenience sample, as Anova Health Institute is the designated United States Agency for International Development support partner in these districts. Urban Johannesburg district is very densely populated, with 3162.1 persons $/ \mathrm{km}^{2}$, compared with 236.0, 61.7 and 61.2 persons/ $\mathrm{km}^{2}$ in Sedibeng, Capricorn and Mopani, respectively. ${ }^{9}$ Medical scheme coverage is highest in Johannesburg (22.2\%), followed by Sedibeng (20.8\%), Capricorn $(8.3 \%)$ and Mopani (6.8\%). ${ }^{9}$ Antenatal HIV prevalence, an indicator of overall population prevalence, was $34.1 \%$ in Sedibeng, $30.9 \%$ in Johannesburg, $26.6 \%$ in Mopani and 22.5\% in Capricorn in $2017 .{ }^{10}$

Records from TIER.Net were included in the analysis for adult PLHIV aged 15-80 years who had initiated ART from 2004 onwards and had been on ART for a minimum of 12 months ( $n=1224366)$. Records were excluded where there were data quality concerns regarding CD4 testing, namely counts $\leq 0$ or $\geq 2000$ cells $/ \mathrm{mm}^{3}$ and nonsensical testing dates $(n=722)$. Records with nonsensical VL testing dates were also excluded $(n=9)$, as were records from facilities which had not exclusively used TIER.Net as their ART management tool, resulting in incomplete TIER.Net data $(n=45445)$. The final data set comprised 1178190 records - 673606 from Johannesburg, 160607 from Sedibeng, 162020 from Capricorn and 181957 from Mopani.

\section{Statistical analysis}

The proportion of patients with a baseline CD4 test and CD4 test subsequent to baseline was calculated, the former was for patients newly initiating ART and the latter was for all patients in the cohort (both new initiators and re-initiators). Post-baseline CD4 tests were defined as CD4 tests performed more than 3 months after ART where the CD4 count was not a duplicate of the baseline count. The proportion of patients with post-baseline CD4 counts $\leq 200$ and $\leq 350$ cells $/ \mathrm{mm}^{3}$ was calculated and compared between different ART durations using a chi-squared test.

Immunological non-responders were defined as patients who had been on ART for more than 4 years and were virally suppressed ( $\mathrm{VL}<1000$ copies $/ \mathrm{mL}$ ) with a CD4 count $\leq 350$ cell $/ \mathrm{mm}^{3}$ based on CD4 testing performed 1 year before to 2 years after the VL test. Time on ART for all patients, including those receiving second- or third-line ART regimens, was calculated as overall time on treatment. Mixed effects logistic regression was used to assess characteristics of INRs, adjusting for random effects at the district level. Fixed effects covariates included ART regimen (first-line, second-line or third-line), age at ART start, gender, baseline CD4 and tuberculosis (TB) status. $p<0.05$ was considered significant.

\section{Ethical consideration}

The study was approved by the Human Sciences Research Council Research Ethics Committee (REC 3/22/08/18). Individual patient consent was not required, as no data were collected for the purposes of this study. Anonymised TIER. Net data that were routinely collected at healthcare facilities for monitoring purposes were used.

\section{Results}

\section{Implementation of CD4 testing}

Baseline CD4 count was recorded for $80.9 \%$ of the 869571 patients newly initiating ART. Amongst all 1178190 adults on ART, only $46.5 \%$ had a CD4 count recorded subsequent to baseline. Amongst patients who had been on ART for 12-18 months $(n=56181)$, only $21.9 \%$ had a post-baseline CD4 test on record.

\section{Low CD4 counts}

Amongst all patients with a baseline CD4 $(n=703869), 50.3 \%$ had counts $\leq 200$ cells $/ \mathrm{mm}^{3}$ and amongst those starting ART since $2017,37.2 \%$ had a baseline count $\leq 200$ cells $/ \mathrm{mm}^{3}$. CD4 count decreased after baseline in $11.0 \%$ of the 443443 patients with both a baseline and subsequent CD4. Amongst all patients with a CD4 test performed after ART start, 14.3\% $(n=78494)$ had a CD 4 count $\leq 200$ cells $/ \mathrm{mm}^{3}$. This proportion was highest amongst patients on ART $\leq 2$ years $(19.7 \%)$ compared with longer treatment durations $(14.8 \%, 14.2 \%$ and $13.8 \%$ amongst patients on ART 2-4, $4-6$ and $>6$ years, respectively, $p<0.001)$.

Just over one-third of patients with a post-baseline CD4 test had a CD4 count $\leq 350$ cells $/ \mathrm{mm}^{3}(35.5 \%, n=194140)$. This proportion was highest amongst patients on ART $\leq 2$ years $(43.9 \%)$ compared with longer durations $(36.1 \%, 35.2 \%$ and $34.7 \%$ amongst those on ART 2-4, 4-6, and > 6 years, respectively, $p<0.001)$. 


\section{Immunological non-responders}

Amongst virally suppressed patients on ART for more than 4 years, $20.0 \%$ ( $n=18$ 556) were INRs. Median CD4 count amongst INRs was 259 cell $/ \mathrm{mm}^{3}$ (interquartile range 127), with $29.5 \%$ having a CD4 count $\leq 200$ cells $/ \mathrm{mm}^{3}$. Immunological non-response was significantly more likely amongst second- or third-line ART patients compared with first-line (odds ratio [OR] 1.85 and 1.54, respectively), amongst older patients compared with those aged 15-25 years (OR 1.12, 1.49 and 1.93 in patients aged 25-35, 35-45 and $\geq 45$ years, respectively), in males (OR 2.45) and amongst patients with TB(OR3.57; Table 1). The odds of immunological non-response were significantly lower amongst patients with baseline CD4 > 350 cells $/ \mathrm{mm}^{3}$ compared with CD4 $\leq 350$ (OR 0.32). In multivariate analysis, INR remained significantly more likely amongst patients on second-line ART (adjusted OR [aOR] 1.79), those aged $35-45$ and $\geq 45$ years (aOR 1.15 and 1.50, respectively), males (aOR 2.28) and patients with confirmed TB (aOR 2.49). The odds of INR also remained significantly less likely amongst patients with higher baseline CD4 (aOR 0.35).

\section{Discussion}

This study of routine data from four South African districts demonstrates poor implementation of CD4 testing subsequent to ART initiation and a notable proportion of patients with low CD4 counts even after initiating ART. Amongst patients on ART for 12-18 months who should have received a routine 12-month CD4 test according to national guidelines, ${ }^{8}$ less than one quarter had a post-baseline
CD4 test recorded. Considering that amongst those who did access CD4 testing, CD4 count was below 200 cells $/ \mathrm{mm}^{3}$ in $20 \%$ of patients on ART $\leq 2$ years, a considerable number of patients with low CD4 counts are being missed. These patients would not receive ACC interventions, including CPT, which may contribute to higher morbidity and mortality.

Immunological non-response was found in $20.0 \%$ of virally suppressed patients in this cohort, in line with reported rates of $10 \%-40 \% .3,5,7$ There is an ongoing need for CD4 count monitoring subsequent to ART initiation in order to identify INRs who require extra clinical care. Efforts should focus on older patients, males and those starting ART with low baseline CD4, as has been previously demonstrated, ${ }^{3,4,5,6,7}$ as well as PLHIV with TB coinfection. It is concerning that low baseline CD4 is significantly associated with immunological non-response, as a notable proportion of patients initiating ART in South Africa do so with low CD4 counts. ${ }^{2}$ In particular, older patients and males are more likely to present late for HIV care with low baseline $\mathrm{CD} 4{ }^{2}$ which may contribute to the poor CD4 recovery of these patients. Continued engagement with communities, particularly with men and older clients, is required to emphasise the importance of engaging with ART services before CD4 counts have dropped substantially. The association between INR and second- or third-line regimens may be because of prolonged viral nonsuppression associated with treatment failure. It is possible that low CD4 counts are a result of delayed switching from a failing to effective regimen, which would need to be further investigated.

TABLE 1: Characteristics of immunological non-responders (CD4 count $\leq 350$ cells $/ \mathrm{mm}^{3}$ ) compared with immunological responders (CD4 count $>350$ cells/mm ${ }^{3}$ ) amongst patients with a suppressed viral load who had been on ART for more than 4 years.

\begin{tabular}{|c|c|c|c|c|c|c|c|c|}
\hline \multirow[t]{2}{*}{ Variables } & \multicolumn{2}{|c|}{$\begin{array}{l}\text { Immunological non-responder, } \\
\qquad n=18556\end{array}$} & \multicolumn{2}{|c|}{$\begin{array}{c}\text { Immunological responder, } \\
n=74134\end{array}$} & \multicolumn{2}{|c|}{ Unadjusted OR } & \multicolumn{2}{|c|}{ Adjusted OR $\dagger$} \\
\hline & $n$ & $\%$ & $n$ & $\%$ & OR $(95 \% \mathrm{Cl})$ & $p$-value & OR $(95 \% \mathrm{Cl})$ & $p$ \\
\hline \multicolumn{9}{|l|}{ Last ART regimen } \\
\hline First line & 13615 & 18.3 & 60709 & 81.7 & Ref & & Ref & \\
\hline Second line & 2208 & 29.5 & 5282 & 70.5 & $1.85(1.76-1.96)$ & $<0.001$ & $1.79(1.68-1.91)$ & $<0.001$ \\
\hline Third line & 34 & 26.6 & 94 & 73.4 & $1.54(1.04-2.28)$ & 0.031 & $1.41(0.85-2.35)$ & 0.188 \\
\hline Unassigned & 22 & 22.5 & 76 & 77.6 & $1.22(0.76-1.97)$ & 0.403 & $0.83(0.44-1.59)$ & 0.581 \\
\hline \multicolumn{9}{|c|}{ Age at ART start, years } \\
\hline $15-25$ & 1104 & 15.4 & 6073 & 84.6 & Ref & & Ref & \\
\hline $25-35$ & 5836 & 16.9 & 28748 & 83.1 & $1.12(1.04-1.20)$ & 0.001 & $0.99(0.90-1.09)$ & 0.886 \\
\hline $35-45$ & 6662 & 21.1 & 24851 & 78.9 & $1.49(1.39-1.60)$ & $<0.001$ & $1.15(1.05-1.27)$ & 0.003 \\
\hline$\geq 45$ & 4954 & 25.5 & 14462 & 74.5 & $1.93(1.80-2.08)$ & $<0.001$ & $1.50(1.37-1.66)$ & $<0.001$ \\
\hline \multicolumn{9}{|l|}{ Gender } \\
\hline Female & 9902 & 15.3 & 54749 & 84.7 & Ref & & Ref & \\
\hline Male & 8654 & 30.9 & 19385 & 69.1 & $2.45(2.37-2.53)$ & $<0.001$ & $2.28(2.19-2.38)$ & $<0.001$ \\
\hline$\leq 350$ & 13831 & 21.6 & 50266 & 78.4 & Ref & & Ref & \\
\hline$>350$ & 664 & 8.0 & 7669 & 92.0 & $0.32(0.29-0.34)$ & $<0.001$ & $0.35(0.32-0.38)$ & $<0.001$ \\
\hline \multicolumn{9}{|l|}{ TB status } \\
\hline None/unknown & 18393 & 19.9 & 73955 & 80.1 & Ref & & Ref & \\
\hline Confirmed TB & 163 & 47.7 & 179 & 52.3 & $3.57(2.88-4.42)$ & $<0.001$ & $2.49(1.81-3.43)$ & $<0.001$ \\
\hline
\end{tabular}

ART, antiretroviral therapy; $\mathrm{Cl}$, confidence interval; OR, odds ratio; Ref, reference; TB, tuberculosis.

Data are $n(\%)$. Total value differs between variables because of missing data.

Statistically significant differences are shown in bold.

$\dagger$, Mixed effects logistic regression for characteristics of immunological non-responders, adjusting for random effects at the district level. Fixed effects covariates include ART regimen level, age at ART start, gender, baseline CD4 count and TB status. 
The strength of this study is the large sample size from a routine patient-level data set that includes clinical variables. However, there are a number of limitations. Firstly, a postbaseline CD4 test was missing in more than half the cohort, which may have introduced bias into the estimation of INRs. This estimation may also have been somewhat inflated by the inclusion of CD4 tests performed up to 2 years after the VL test. Secondly, we did not have access to all post-baseline CD4 and VL tests; only the most recent result was available. We could, therefore, not assess change in CD4 count over time following ART initiation, nor could we assess timing of viral suppression amongst patients with treatment failure who were switched to a second- or third-line regimen, which may have impacted time to CD4 recovery. Thirdly, we only had access to CD4 test results that had been captured into TIER.Net. CD4 counts that were missing from TIER.Net could, therefore, not be included in the analysis. It is unclear whether missing CD4 data represent poor implementation of CD4 testing in clinical practice or a data capturing problem, and this warrants further investigation. Finally, although we believe the study sample to be generally representative of the South African population as it includes both urban and rural areas, as well as districts with high and low socio-economic status, findings should be extrapolated to other areas with caution, particularly because this was not a random sample.

In conclusion, it is essential that healthcare workers are educated regarding the ongoing importance of correctly implementing CD4 testing guidelines in the universal test and treat era to identify patients with low CD4 counts who require extra clinical care. Quality of care needs to be improved and existing ACC guidelines more widely implemented in order to support improved clinical outcomes in patients with low $\mathrm{CD} 4$ counts.

\section{Acknowledgements}

The authors would like to gratefully acknowledge Michel Muteba who assisted with the statistical analyses.

\section{Competing interests}

The authors have declared that no competing interests exist.

\section{Authors' contributions}

K.R. conceived the study. R.R.L. cleaned the data and performed the analysis. N.D., L.G. and K.R. assisted with the interpretation of the results. R.R.L. drafted the manuscript. J.A.M. and H.E.S. supervised the project and obtained the funding. All authors critically reviewed and edited the manuscript and gave their consent for publication.

\section{Funding information}

This study is made possible by the generous support of the American people through the US President's Emergency Plan for AIDS Relief (PEPFAR) through the United States Agency for International Development (USAID) under Cooperative Agreement number 72067418CA00023 for the Accelerating Program Achievements to Control the Epidemic (APACE) Activity in South Africa to the Anova Health Institute. The contents are the responsibility of Anova Health Institute and do not necessarily reflect the views of USAID or the United States Government. The funders had no role in study design, data collection and analysis, decision to publish or preparation of the manuscript.

\section{Data availability statement}

The data that support the findings of this study are available on request from the corresponding author K.R. The data are not publicly available because they contain patient-level HIV information that could breach patient confidentiality.

\section{Disclaimer}

The views and opinions expressed in this article are those of the authors and do not necessarily reflect the official policy or position of any affiliated agency of the authors.

\section{References}

1. World Health Organization. Guidelines for managing advanced HIV disease and rapid initiation of antiretroviral therapy. Geneva: World Health Organization; 2017.

2. Lilian RR, Rees $\mathrm{K}$, Mabitsi M, et al. Baseline CD4 and mortality trends in the South African human immunodeficiency virus programme: Analysis of routine data. South
Afr J HIV Med. 2019;20(1):963. https://doi.org/10.4102/sajhivmed.v20i1.963

3. Asmelash A, Zheng $Y$, Kaloustian KW, et al. Predictors of suboptimal CD4 response among women achieving virologic suppression in a randomized antiretrovira treatment trial, Africa. BMC Infect Dis. 2014;14:331. https://doi. treatment trial, Africa. BN

4. Balachandra S, Rogers JH, Ruangtragool L, et al. Concurrent advanced HIV disease and viral load suppression in a high-burden setting: Findings from the 2015-6 ZIMPHIA survey. PLoS One. 2020;15(6):e0230205. https://doi.org/10.1371/ journal.pone.0230205

5. Boatman JA, Baker JV, Emery S, et al. Risk factors for low CD4+ count recovery despite viral suppression among participants initiating antiretroviral treatment with CD4+ counts > 500 cells $/ \mathrm{mm}^{3}$ : findings from the Strategic Timing of AntiRetroviral Therapy (START) trial. J Acquir Immune Defic Syndr. 2019;81(1) 10-17. https://doi.org/10.1097/QAl.0000000000001967

6. Gunda DW, Kilonzo SB, Kamugisha E, et al. Prevalence and risk factors of poor immune recovery among adult HIV patients attending care and treatment centre in northwestern Tanzania following the use of highly active antiretroviral therapy: A retrospective study. BMC Res Notes. 2017;10(1):197. https://doi.org/10.1186/ s13104-017-2521-0

7. Engsig FN, Gerstoft J, Kronborg G, et al. Long-term mortality in HIV patients virally suppressed for more than three years with incomplete CD4 recovery: A cohort study. BMC Infect Dis. 2010;10:318. https://doi.org/10.1186/1471-2334-10-318

8. National Department of Health of South Africa. National consolidated guidelines for the management of HIV in adults, adolescents, children and infants and prevention of mother-to-child transmission. Pretoria: NDoH; 2020.

9. Massyn N, Barron P, Day C, et al., editors. District health barometer 2018/19. Durban: Health Systems Trust; 2020.

10. Woldesenbet SA, Kufa T, Lombard C, et al. The 2017 National antenatal sentinel HIV survey key findings, South Africa. Pretoria: National Department of Health; 2019. 